\title{
Efectos de la violencia en los procesos de historización: mediación simbólica, vínculo social y singularidad
}

\section{The effects of violence on historization processes: symbolic mediation, social ties and singularity}

Daniel Jofré*1

El artículo se interroga sobre los efectos de la violencia en los procesos subjetivos de historización y en el vínculo social. Con este objetivo se desarrolla un estudio teórico que integra reflexiones psicoanalíticas e hipótesis clínicas y filosóficas sobre la violencia en las sociedades contemporáneas. Se sostiene que la elaboración de los efectos de la violencia depende de la construcción de las mediaciones simbólicas que permiten la inscripción y singularización de la historia subjetiva en el vínculo social.

Palabras clave: Violencia, historización, orden simbólico, singularidad

${ }^{* 1}$ Universidad Austral de Chile (Puerto Montt, Chile). 


\section{Introducción}

El estudio de los fenómenos de violencia y su impacto en los procesos de subjetivación e institución de los vínculos sociales (Aquatias, 1997; Bourdieu, 1986; Dubet, 1992; Foucault, 1979; Héritier, 2001; Segato, 2003), requiere dar cuenta de la disposición de la violencia de persistir como efecto traumático o de generar formas de vínculo social. En esta óptica, el problema de la violencia recubre el evento agresivo, pero también el momento de creación de instituciones sociales y el espacio de las conmemoraciones simbólicas de los pueblos, lo que introduce el análisis de estos fenómenos en los procesos que permiten interpretar la historia de las sociedades humanas. No obstante, la dilucidación de la lógica que aloja la temporalidad de los fenómenos de violencia resulta problemática o a lo menos de difícil discernimiento.

La aproximación psicoanalítica al problema de la violencia es indisociable de la reflexión de Freud sobre el sufrimiento humano y sobre los cuestionamientos éticos que conlleva procurar sobrellevarlo. Freud analizará el problema de la violencia intentando alejarse de todo naturalismo - que comporta pensar la violencia anclada sólo en los soportes biológicos del ser humano - y de todo idealismo - que supusiera una posible resolución definitiva de la violencia. Freud introduce el problema de la violencia en la lógica con que piensa el sufrimiento humano, discerniendo por tanto sus efectos y sus posibilidades de elaboración en la temporalidad diacrónica que se introduce entre el impacto agresivo y su repetición. La inclusión de esta lógica temporal acarrea algunas interrogantes respecto de las coordenadas intrapsíquicas en que se establece el registro y la elaboración de los eventos traumáticos, como también, implica un desarrollo teórico capaz de articular los planos individual y colectivo, en una aproximación que debe dar cuenta tanto de la capacidad de la violencia de perturbar el psiquismo individual, como de su potencialidad de impactar en la construcción de las representaciones sociales que conforman la memoria colectiva.

En este marco, la pregunta que guía nuestro estudio busca ponderar la importancia de las mediaciones simbólicas que permiten la inscripción del sujeto en el vínculo social, en la elaboración subjetiva de los efectos de la violencia. Con este objeto, se estudian los desarrollos de Freud sobre 


\section{ARTIGOS}

la demanda de apropiación de la violencia originaria, como elemento integrante de los procesos que deben realizar los individuos y las colectividades para asegurar el traspaso generacional e instituir el vínculo social; así como también, se estudian fuentes concernientes a los efectos de la violencia extrema y al problema de la generalización de la violencia en las sociedades contemporáneas. Nuestra hipótesis sitúa el espacio abierto por las mediaciones simbólicas que instituyen lo humano, como el resorte fundamental que permiten los procesos de historización y de singularización frente a la violencia.

La argumentación posee dos partes: i) Violencia y trabajo de transmisión intergeneracional, y, ii) Discusión: historización y singularidad como respuesta a la violencia. En el primer título se examinan las mediaciones intrapsíquicas e interpsíquicas que permiten el proceso de transmisión transgeneracional, tomando como eje el estudio de la relación entre el sujeto, el superyó y el Ideal del yo. En segundo lugar, se analizan las dificultades e impedimentos a este trabajo de transmisión psíquica, examinando dos hipótesis sobre situaciones de violencia extrema: la noción de melancolización del lazo social (Douville, 2001) y de Espacio entre las generaciones (Uwineza \& Brackelaire, 2014). La segunda parte del artículo tiene por objeto desarrollar algunas reflexiones sobre la importancia de los procesos de historización frente a la violencia generalizada de las sociedades contemporáneas, tomando como eje los procesos de singularización y reconocimiento simbólico. Esta reflexión busca contraponer los efectos de los eventos de violencia - extrema y generalizada - y los procesos de historización intergeneracional y de construcción identitaria.

\section{Primera Parte \\ Violencia y trabajo generacional}

\section{Desarrollos freudianos sobre la violencia, formaciones de masa y transmisión psíquica}

Dentro de las reflexiones de Freud, la forma de abordar la cuestión de la violencia es indisociable de una reflexión sobre el vínculo entre el hombre, el sostén pulsional de sus acciones y la cultura. En el mito del Padre originario propuesto por Freud (1914/1978a), se representa los orígenes de la convivencia cultural de los hombres como consecuencia de una violencia originaria, seguida por la instauración de las reglamentaciones de la vida colectiva. La 
ambivalencia ante la Ley, la culpabilidad y la hostilidad ante los semejantes constituyen, en el marco de esta reflexión, la expresión psíquica resultante del intento de apaciguar la arbitrariedad de la violencia del Padre originario, reemplazándola por una violencia institucionalizada, es decir, por la fuerza del derecho y por las imposiciones contenidas en los ideales de la vida colectiva.

En este sentido, al interior del esquema de análisis desarrollado por Freud, la figura del superyó será a la vez, depositaria de la arbitrariedad de la Ley y de las disposiciones e ideales colectivos que conforman la identidad cultural de los pueblos (Freud, 1940/1978e). Se integra, por tanto, en la instancia psíquica del superyó la historia individual y la historia colectiva. De hecho, en el intento por comprender el origen de la severidad del sentimiento de culpabilidad en el niño Freud introduce de manera complementaria, al infante en su propia vida, en la historia de su cultura y en la historia de la humanidad. El carácter complementario de estas fuerzas que operan sobre el sujeto no hacen posible proceder a su desagregación. Al contrario, se tratará más bien, de visualizar la superposición de los procesos históricos instituyentes de las representaciones culturales e ideales colectivos y los procesos de subjetivación del niño frente a las exigencias del superyó.

La intuición de esta superposición de estratos psíquicos y culturales permite situar a los procesos identificatorios constituyentes del psiquismo infantil en la intersección del padre y el Padre; como también permite reconocer el vínculo entre el mundo fantasmático infantil y el horizonte mítico de los pueblos y de la humanidad. En esta perspectiva, en la Obra de Freud (1929/1978c), las leyes de los pueblos hacen re-emerger las posiciones subjetivas presentes dentro del mito originario, permitiendo visualizar una expresión de la condición humana ligada a la culpabilidad y a la lucha pulsional, la cual se manifestaría aún en la actualidad en las dificultades de los hombres en sus actividades de crianza, educación o coexistencia.

Empero, una segunda veta de los análisis freudianos sobre la violencia se encuentra en los desarrollos que Freud presenta en "Psicología del yo y análisis de las masas" (1921/1978b). Estas elaboraciones permiten introducir nuevas luces sobre los postulados psicoanalíticos sobre la violencia y la lógica de relaciones que el sujeto establece respecto de las instancias psíquicas ideales y el superyó. De hecho, las líneas dedicadas por Freud al análisis de las masas explicitan el empobrecimiento de los procesos psicológicos, tanto conscientes como inconscientes, que ponen freno a las pulsiones más primitivas. Este empobrecimiento de la vida psíquica suspende todas las adquisiciones que ha forjado el trabajo de la cultura en los individuos. En este sentido, se puede subrayar el carácter ambivalente del rasgo identificatorio 


\section{ARTIGOS}

propuesto por el líder, en cuanto, de carácter imaginario y narcisístico, revive la complacencia imaginaria del Yo Ideal, rehusando, pese a ser un fenómeno colectivo, las mediaciones psíquicas e interpsíquicas que operan para lograr el diferimiento de la vida pulsional. Dejando ver que, desde el punto de vista de los procesos de elaboración del impulso agresivo, la apelación a un orden "exterior" al individuo (la patria, la raza, la tradición) no se vincula necesariamente con la apertura del sujeto a las mediaciones culturales frente a la vida pulsional. De hecho, como Freud lo notara, en dichos imaginarios que se conforman resaltando las pequeñas diferencias, el origen o el destino de los pueblos, anida la violencia y la agresividad frente al otro, resultando ser, por tanto, una vía aún más expedita para la expresión de estas manifestaciones.

Un tercer terreno de las elaboraciones de Freud que permite pensar la relación entre la violencia y el vínculo social, se halla en "Moisés y la religión monoteísta" (1939/1978d). En este trabajo Freud desarrolla algunas observaciones de interés sobre la articulación entre la construcción de ideales en los pueblos y la culpabilidad como expresión de los mecanismos psíquicos operantes en el superyó. En este sentido, cabe remarcar que la institución del Padre primordial como ideal del grupo de hermanos, adquiere en este análisis, el carácter de la formación de una alianza intergeneracional: aquella del Padre primordial y sus hijos parricidas, pero que igualmente incluye a los están por venir, es decir, a las generaciones de los descendientes que deben tramitar la deuda adquirida.

Alain Badiou (2010), observa en la construcción freudiana sobre los procesos de transmisión psíquica, la edificación de un esquema dialéctico que articula el estado inmediato y violento de la agresividad, el estado simbólico de la sumisión a la ley, $y$, el estado final de amor compartido; de modo que, el amor finalmente reemplaza a la muerte por la mediación de la Ley. En este esquema, que permite la construcción identitaria de los sujetos y la elaboración transgeneracional de la violencia, las figuras de la deuda y la reconciliación, aparecen como los resortes fundamentales de la lógica que articula las acciones recíprocas que permiten el traspaso generacional. Pero también, estas figuras permiten la mediación entre el ideal y el superyó, articulando la historia individual y la colectiva. Por consiguiente, el trabajo psíquico de apropiación necesario para sobrellevar la violencia, adquiere el carácter de una mediación simbólica tendiente a apaciguar las exigencias superyoicas y los ideales narcisistas, en virtud de la identificación del sujeto a la lógica que organiza el traspaso generacional y promueve los procesos sublimatorios.

En síntesis, la aproximación freudiana permite visualizar un modo de adentrarse al problema de la violencia que no se agota en la comprensión de los 
diferentes aspectos, sociohistóricos o individuales que intervienen en ella. Sino que intenta construir frente al problema de la violencia un esquema interpretativo propiamente metapsicológico, haciendo posible establecer: i) la ausencia o la debilidad de las mediaciones subjetivas (intrapsíquicas e interpsíquicas) constitutivas de la dialéctica de la palabra y el deseo, sitúa al individuo como una mera reacción frente a los dispositivos ideales, el superyó y las disposiciones agresivas constitutivas del psiquismo, y, ii) la construcción dialéctica de la subjetividad permite reconocer el entrecruzamiento generacional, como una modalidad de apropiación de los acontecimientos de violencia (no sólo como verdad material sino especialmente en tanto que construcción histórico-vivencial) constitutivos y generativos de formas de vinculación social.

\section{Hipótesis contemporáneas sobre situaciones de violencia extrema: traumatismo y el impasse de los procesos de historización y transmisión}

Con el objeto de profundizar en el análisis y dilucidación del modo en que se elaboran simbólica e históricamente los efectos de violencia, en este apartado, se revisarán brevemente dos modelos interpretativos que, vinculando presupuestos antropológicos y clínicos, permiten visualizar la ruptura de las mediaciones simbólicas producto de la violencia extrema. El principal objetivo de esta sección será intentar desentrañar cómo la violencia extrema puede romper con la temporalidad lógica que introducen las mediaciones psíquicas e interpsíquicas vislumbradas por Freud, pero también atisbar cómo opera la regeneración de estos vínculos que confieren consistencia a la condición humana y a la vida social frente a la violencia extrema.

Primero, el impacto de la violencia en los procesos de subjetivación: la apuesta por construir una aproximación antropológica y clínica frente a situaciones sociales de conflicto extremo permite comprender el modo en que se articula la historia individual a los procesos simbólicos que organizan la memoria colectiva. Así, a partir del caso de los niños y adolescentes soldados de las guerras africanas - en donde la violencia aspira no sólo derrocar sino exterminar al otro - Olivier Douville, $(2013$; 2016) remarca: i) la depreciación del enemigo hasta convertirlo en una cosa, los asesinatos de padres u otros familiares como iniciación a la lógica de una guerra sin fin, la suspensión de los rituales mortuorios - que implica la depreciación de la diferencia entre la vida y la muerte; constituyen formas de ruptura del horizonte simbólico. ii) $\mathrm{La}$ pérdida de vínculos referenciales al semejante y a los ancestros, reduce al sujeto 


\section{ARTIGOS}

a una constante demanda imaginaria que se encierra sobre pequeños grupos de referencia que promueven ideales de fragmentación de los vínculos fraternales con el otro. A partir de estas indicaciones, se hace posible observar: iii) la emergencia de un afecto demostrativo de un profundo desamparo y vergüenza, que expresa la desatadura del horizonte subjetivo respecto de los apuntalamientos simbólicos que organizan los intercambios sociales.

Según esta hipótesis, en el centro de este estado de melancolización del lazo social y de retraimiento subjetivo se expresa el triunfo de las exigencias sádicas del superyó sobre la vida psíquica. De suerte que, será el carácter sádico del superyó, el que retorne sobre el sujeto aquietando la emergencia del deseo a cambio de su identificación a ideales guerreros, en lo esencial, vacíos de sentido. Así, el triunfo de la violencia representa también el impasse del trabajo de elaboración transgeneracional, en cuanto supone, la suspensión de la inscripción de la vida psíquica individual dentro del horizonte simbólico que modula el trabajo generacional y posibilita el encuentro con el otro.

Segundo, re-generación de lo humano frente a la violencia extrema: las relaciones que son forjadas por eventos de violencia masiva son objeto actualmente de una serie de investigaciones antropológicas, psicológicas y médicas que buscan comprender el impacto de la violencia extrema en la salud física y mental, en la relaciones familiares o comunitarias, y en la conformación del sentimiento de unicidad psíquica e identidad social (Altounian, 2013; Cadoret, 2003; Evans-Campbell, 2008; Giot, 2013; Mitchell \& Maracle, 2005; Kirmayer, Brass \& Tait, 2000). En este contexto, siguiendo un enfoque multidisciplinario Jeannette Uwineza y Jean Luc Brackelaire (2014), desarrollan una reflexión sobre los modos en que se instituye y comparte el espacio entre las generaciones, a partir de experiencias recogidas durante intervenciones clínicas con víctimas sobrevivientes del genocidio de Ruanda.

De acuerdo a estos investigadores, este espacio entre las generaciones busca reconstruir los lazos entre los sujetos fracturados por las experiencias de violencia, favoreciendo, por lo tanto, la apropiación de la herencia transgeneracional. Se define, en este sentido, la construcción del espacio entre las generaciones, como un proceso que compromete un trabajo y una operación de apropiación trasformadora y creadora de sí mismo y del otro, que implica desarrollar una serie de elaboraciones que permiten la desapropiación y la reapropiación de aquello que es transmitido por el trauma: usurpación, abuso, aniquilación. Por consecuencia, la noción de lo transgeneracional no designa para estos autores los contenidos, narraciones o historias que se pueden o no transmitir, tampoco designa los medios en que se puede desarrollar este 
pasaje, es decir, cómo se transmite el pasado traumático. Fundamentado en el modelo dialéctico de la Persona (Gagnepain, 1991), los procesos psíquicos e interpsíquicos que se despliegan en el espacio entre las generaciones suponen una serie de demarcaciones e investimentos positivos, que, en último término, conforman el proceso subyacente e implícito de delimitación generacional y de conformación de relaciones bilaterales de responsabilidad y obligación respecto de otros individuos.

De este modo, la elaboración del espacio entre las generaciones después de situaciones extremas de violencia tiene por objeto restituir el horizonte simbólico perdido durante estas experiencias. En este espacio, se ponen en juego figuraciones y discursos expresivos de formas personales de apropiación de la herencia transgeneracional, capaces de reorganizar y reestructurar los posicionamientos individuales frente al orden social y la historia colectiva. Siendo, por tanto, una forma de cuestionar la herencia traumática de una violencia que tuvo como objetivo desmoronar las raíces mismas que permiten la institución de lo humano en el registro de los vínculos simbólicos de obligación, reciprocidad y responsabilidad. Razón por la cual, este proceso de reapropiación conlleva momentos de vivos cuestionamientos sobre aquello que es heredado y sobre aquello que constituye los pilares de la propia identidad social, siendo generativo, por tanto, de un trabajo de apropiación de los códigos sociales y la historia colectiva.

\section{Segunda Parte: \\ Discusión}

\section{Violencia, historización y singularidad}

La violencia extrema constituye un ámbito de análisis privilegiado en donde es posible comprender - en cuanto lo amplifica - el impacto deshumanizante de la violencia, pero también, la lógica que permite su elaboración. Se estima que la posibilidad de elaboración de la violencia extrema se relaciona con la institución del hombre de acuerdo a las mediaciones simbólicas, entre las cuales se cuentan aquellas del reconocimiento y la alianza entre las generaciones. Sin embargo, esta violencia extrema que es posible reconocer en genocidios y otros episodios de exterminio, no es completamente ajena a la violencia que se generaliza en los modos de relación social presentes en las sociedades contemporáneas, de suerte que es admisible 


\section{ARTIGOS}

abrir nuestra discusión a otros modos de expresión de las lógicas simbólicas con que la humanidad responde a la violencia.

Bertrand Ogilvie (2012) observa que, en la actualidad, la configuración de un modo de violencia cualitativamente diferente a la de las sociedades modernas. Esta violencia cotidiana expresa la desligadura fundamental de las relaciones humanas que es instituida por las mutaciones sociales que introduce el capitalismo, en su faceta más acelerada y extrema. De acuerdo a este autor, en las sociedades contemporáneas es posible reconocer la generación de individuos insertos en formas de intercambio y de exclusión que, en último término tornan a estos sujetos, desechables. Se instala así una lógica de exterminación indirecta o delegada, que produce masas de individuos excluidos - o en vías de exclusión - de toda forma de reconocimiento simbólico. En esta violencia contemporánea, el sujeto se confronta a su propia destrucción, $o$, en otros términos, a la imposibilidad de su representación e institución. De suerte que es posible señalar que, esta violencia ordinaria interroga los modos en que los actuales vínculos sociales generan formas de impedimento para la emergencia de la subjetividad.

Esta cuestión, no es ajena del todo a las teorizaciones psicoanalíticas sobre las dificultades presentes en la edificación subjetiva dentro de las sociedades contemporáneas, puesto que en éstas también se interroga las posibilidades de conformación del sujeto, en ausencia de la lógica terciaria que introduce el reconocimiento por el Otro y la identificación simbólica (Sciara, 2007). De hecho, en estas indagaciones se repara en que producto del aumento de mecanismos de interacción social profundamente segregativos, se refuerza una lógica esencialmente dual e imaginaria que se consolida en la generación de espacios de marginalización y rechazo de la alteridad. Caroline Guendouz-Barbaras (2009) observa que la generación de espacios de exclusión y de segregación presente en las prácticas sociales instituidas por el discurso capitalista - en particular en su faceta neoliberal —, terminan por redoblar las alienaciones imaginarias del sujeto, procurando así un emplazamiento social a la figura del individuo. De esta manera, para estos autores, en el punto de confluencia entre estructuración simbólica del sujeto y discurso capitalista, el vínculo social vigente en las sociedades de consumo masificado tiende a impedir los procesos psíquicos y las mediaciones sociales que permiten la edificación del sujeto según la lógica que organiza el orden simbólico:

Todo indica que el discurso capitalista sustituye a una organización vertical fundada por el al-menos uno (el Padre), por la horizontalidad de la red cuya tela a recorrer es el caldo de una a-cultura de la errancia. La horizontalidad 
segrega porque ella prohíbe toda inscripción en un lugar y, por consecuencia, aísla en ninguna parte: ella se desarrolla en relaciones de lateralidad, en red, agregados cuyo lazo no es sino del orden del collage, de la adherencia. (2009, p. 164; traducción personal)

Se trataría, por consiguiente, de la consolidación de prácticas e interacciones sociales que en ausencia de una referencia simbólica de vectorización, instituyen formas de horizontalidad que masifican las posiciones subjetivas frente a la alteridad y el deseo. Así comprendido, el vínculo social capitalista genera una violencia que produce modos imaginarios de reconocimiento, destinaciones subjetivas que reniegan de la temporalidad lógica que hacen intervenir las mediaciones simbólicas, a cambio de la inmediatez erigida por lo imaginario. En la línea de estas conjeturas se advierte (Dufour, 2003) que el vínculo social contemporáneo es capaz de forjar una búsqueda identificatoria que acelera la alienación a retóricas sociales como la religión, la comunidad o la etnia.

A partir de lo anterior, sería posible sostener que el peligro de la violencia generalizada que se despliega en las sociedades contemporáneas, se encuentra en la proliferación de anclajes identitarios que, mediante la construcción de una ilusión retrospectiva, permiten a sujetos fuertemente precarizados y excluidos, abrazar una imagen que otorga una pertenencia idealizada y añorada.

Ampliemos el análisis: la extrema singularización del orden social contemporáneo (Brackelaire, 2009) conlleva la suspensión de los procesos psíquicos e interpsíquicos constitutivos de la dialéctica de la persona, puesto que reniega no sólo de la construcción de los espacios de inscripción de los individuos en lo social, sino porque también impide el despliegue de los procesos de desapropiación y apropiación subjetiva del orden social instituyente. En este sentido, se puede subrayar como uno de los efectos de la violencia del orden socio discursivo contemporáneo, la alteración de la disposición a la historización y a la dificultad de dar cuenta de una apertura subjetiva a la alteridad dentro del trabajo de construcción identitaria.

Articulando estas perspectivas de análisis sobre la violencia, se podría conjeturar que sus efectos: i) se visualizan en la falta de inscripción del sujeto al vínculo social y en la cadena de las generaciones, y ii) se expresan en la producción de formas de impedimento en la construcción de una identidad social que le permita al individuo posicionarse frente a la historia colectiva, puesto que también condiciona la posibilidad del sujeto de integrar sus renuncias pulsionales a un horizonte simbólico, des-historizando de ese modo la identidad psíquica.

Por tanto, esta dificultad de dar curso a los procesos de historización expondría, a nuestro juicio, una cierta incapacidad de orientar la apropiación subjetiva de la memoria colectiva, pero también el impedimento subjetivo 


\section{ARTIGOS}

frente a la historia fantasmática que narra las renuncias pulsionales, las ligazones y desasimientos que han permitido la construcción de la identidad psíquica. Así, en lugar del trabajo de elaboración psíquica de apropiación del cuerpo y de inscripción generacional, operaría más bien una suerte de desplazamiento de la violencia.

Siguiendo esta hipótesis, podría admitirse que el impasse psíquico y social que provoca la violencia en las sociedades contemporáneas, redobla la ambivalencia frente a la historia personal y la historia colectiva. Esta ambivalencia, de tonalidad superyoica, sería capaz de instalar en el sujeto la necesidad de reconocerse como participe de una trama histórica, familiar y colectiva, pero de un modo en que este horizonte identificatorio no permite desplegar el circuito de la deuda (que conlleva reconocer un horizonte simbólico que ha acogido y que antecede al sujeto) y la responsabilidad (que conlleva comprenderse como participe de vínculos de reciprocidad ante otros sujetos), impidiendo, por tanto, la emergencia de la alteridad.

Se pueden realizar dos puntualizaciones a este respecto. Si el trabajo de inscripción en la cadena de las generaciones y en el vínculo social supone la expresión de una apertura del sujeto a la historia colectiva y sobre sí mismo, el despliegue propiamente humano frente a la violencia consiste en el reencuentro del sujeto con el carácter histórico procesual - y no connatural - de la violencia. En este sentido, la posibilidad de elaborar individual e intersubjetivamente la violencia tendría relación con el reconocimiento de la violencia como participe de la propia condición subjetiva y de la institución misma del orden social, pero también supondría el discernimiento de sus efectos dentro de una matriz histórica habilitada para restituir nuevos posicionamientos subjetivos frente a la violencia. Por otra parte, se puede indicar que los efectos de violencia, la exclusión y el abandono, pueden expresarse como una paralización del trabajo de singularización de los anclajes constituyentes de la subjetividad, propiciando formas de encuentro con el otro, atravesadas por una profunda masificación de las posiciones subjetivas, como es posible reconocer en fenómenos de violencia en donde la sumisión absoluta a ideales grupales o sectarios producen efectos de masa. Se trataría, en este caso también de una violencia que tiende a la homogenización de la vivencia psíquica del sujeto como respuesta a la exclusión, a la instrumentalización de la vida contemporánea y, en definitiva, a la pérdida de consistencia de los lazos simbólicos que conforman lo humano. Así, a contracorriente de los esfuerzos psíquicos de historización y de singularización, el problema de la perpetuación y del acrecentamiento de la violencia responde al impedimento de la articulación del sujeto a la temporalidad lógica instituida por lo inconsciente/implícito, 
obturando de ese modo, el espacio psíquico e intersubjetivo que se abre entre las generaciones y entre los significantes claves del vínculo social.

\section{A modo de conclusión}

Otorgar un rol clave a los mediadores simbólicos que permiten al sujeto explorar la violencia extrema y ordinaria como productos históricos y no como una necesidad o una obligación proveniente de lo real, significa comprender que salir del tiempo de la repetición de la violencia, conlleva también un trabajo de superación de una lógica especular en donde la figura del otro deviene y se consolida como persecutora y agresiva, perpetuando sus efectos transgeneracionalmente y amplificando su impacto a grupos cada vez más numerosos dentro de la sociedad. En este sentido, sería posible concluir que responder a los efectos de violencia conlleva, para el sujeto, reconocerse no sólo como participe de una historia colectiva dentro de una linealidad imaginaria, puesto que también supone la disposición del sujeto a reconocer su herencia y soporte simbólico.

En esta perspectiva, el trabajo de elaboración de la violencia podría ser entendido como un modo de poner de relieve la lógica ternaria que permite distinguir el tiempo del evento (momento del impacto agresivo o de la violencia), el momento de comprender - que conlleva una abstracción del sujeto respecto de la situación o evento - y el momento de concluir o de re-investidura, que implica un reposicionamiento frente a la propia experiencia e historia (momento lógico en donde no cabe aplicar la distinción entre lo individual y lo colectivo). A nuestro juicio, entre estas mediaciones lógicas constitutivas del lazo interhumano y de su transmisión, se abre el espacio del reconocimiento del otro como una necesidad antropológica frente a la violencia, lo que permite por fin, entreabrir el espacio subjetivo a la negatividad inherente a lo humano y a su elaboración histórica y ficcional.

En consecuencia, es posible sostener que comprender los anclajes generacionales y simbólicos frente a la violencia admite las siguientes consideraciones: i) los mecanismos de elaboración de la violencia suponen una lógica transgeneracional que permite la representabilidad de los eventos y situaciones traumáticas, lo cual conlleva reconocer; ii) que el surgimiento ficcional del sujeto, es decir, la emergencia del sujeto como efecto de sentido frente a las retóricas y prácticas de la violencia, resulta de la apropiación del espacio significante que articula la cadena de las generaciones y el vínculo social. 


\section{ARTIGOS}

Pudiéndose admitir, por tanto, iii) que la elaboración de la memoria histórica asociada a procesos de violencia requiere de la apropiación de los anclajes imaginarios de la identidad social mediante su articulación al tiempo lógico que historiza la identidad psíquica; generando un espacio simbólico de traducción y tramitación, de creación y recreación del orden humano, y no sólo de desplazamiento, de los elementos traumáticos de una generación a otra.

\section{Referencias}

Altounian, J. (2013). Remonter le temps, créer l'héritage. In J. L. Brackelaire, M. Cornejo, \& J. Kinable, Violence politique et traumatisme: Processus d'élaboration et de création. Bruxelles: Editions Academia-L'Harmattan.

Aquatias, S. (1997). Jeunes de banlieue, entre communauté et société. Une approche socio-anthropologique du lien social. Socio-anthropologie, (2).

Badiou, A. (2010). Sigmund Freud: Anthropologie de la guerre. Paris, France: Fayard.

Bourdieu, P. (1986). Habitus, code et codification. Actes de la recherche en sciences sociales, 64(1), 40-44.

Brackelaire, J. L. (2009). La personne en suspens. Singularisme moderne et courtscircuits dans la transmission. In Gauchet \& Quentel, Histoire du sujet et théorie de la personne (pp. 123-144). Rennes, France: Presses Universitaires de Rennes.

Cadoret, M. (2003). Le paradigme adolescent. Paris, France: Dunod.

Douville, O. (2001). Pour introduire l'idée d'une mélancolisation du lien social. Cliniques méditerranéennes, 1, 239-262.

Douville, O. (2013). Situations et destinées des enfants et des adolescents dans la guerre en Afrique. Études sur la mort, 2, 55-68.

Douville, O. (2016). De la honte à l'indifférence. Situations d'enfants et d'adolescents... sous la guerre, en Afrique. La clinique lacanienne, 1, 199-212.

Dubet, F. (1992). A propos de la violence et des jeunes. Cultures \& conflits, 2.

Dufour, D. R. (2003). L'art de réduire les têtes: sur la nouvelle servitude de l'homme libéré, à l'ère du capitalisme total. Paris, France: Denoël.

Evans-Campbell, T. (2008). Historical trauma in American Indian/Native Alaska communities: A multilevel framework for exploring impacts on individuals, families, and communities. Journal of interpersonal violence, 23(3), 316-338.

Foucault, M. (1979). Microfísica del poder. Madrid, España: La Piqueta.

Freud, S. (1978a). Tótem y tabú. In J. Strachey (Ed.) y J. L. Etcheverry (Trad.), Obras completas: Sigmund Freud (Vol. XIII). Buenos Aires, Argentina: Amorrortu. (Trabajo original publicado en 1914). 
Freud, S. (1978b). Psicología de las masas y análisis del yo. In J. Strachey (Ed.) y J. L. Etcheverry (Trad.), Obras completas: Sigmund Freud (Vol. XVIII). Buenos Aires, Argentina: Amorrortu. (Trabajo original publicado en 1921).

Freud, S. (1978c). El malestar en la cultura. In J. Strachey (Ed.) y J. L. Etcheverry (Trad.), Obras completas: Sigmund Freud (Vol. XXI). Buenos Aires, Argentina: Amorrortu. (Trabajo original publicado en 1929).

Freud, S. (1978d). Moisés y la religión monoteísta. In J. Strachey (Ed.) y J. L. Etcheverry (Trad.), Obras completas: Sigmund Freud (Vol. XXIII). Buenos Aires, Argentina: Amorrortu. (Trabajo original publicado en 1939).

Freud, S. (1978e). Esquema de psicoanálisis. In J. Strachey (Ed.) y J. L. Etcheverry (Trad.), Obras completas: Sigmund Freud (Vol. XXIII). Buenos Aires, Argentina: Amorrortu. (Trabajo original publicado en 1940).

Gagnepain, J. (1991). Du vouloir dire II. De la personne. De la norme. Paris, France: Livre et communication.

Giot, J. (2013). Traduire, transmettre: ouvres de présences. In J. L. Brackelaire, M. Cornejo, \& J. Kinable, Violence politique et traumatisme: Processus d'élaboration et de création. Bruxelles: Editions Academia-L'Harmattan.

Guendouz-Barbaras, C. (2009). Errance et exclusion dans la subjectivité contemporaine. La clinique lacanienne, 2, 157-167.

Héritier, F. (2001). Les fondements de la violence. Ponencia presentada en el Seminario La Violence, les Mots et le Corps. Paris: IRESCO/GERS. [Links].

Kirmayer, L. J., Brass, G. M., \& Tait, C. L. (2000). The mental health of Aboriginal peoples: Transformations of identity and community. The Canadian Journal of Psychiatry, 45(7), 607-616.

Mitchell, T. L., \& Maracle, D. T. (2005). Post-Traumatic Stress and the Health Status of Aboriginal Populations in Canada. International Journal of Indigenous Health, 2(1), 14-23.

Ogilvie, B. (2012). Violence et représentation. In Ogilvie, L'homme jetable. Essai sur l'exterminisme et la violence extrême (pp. 59-85). Paris, France: Éditions Amsterdam.

Sciara, L. (2007). Les lieux de ségrégation constituent-ils la pointe avancée de la clinique contemporaine? Journal français de psychiatrie, 1, 35-38.

Segato, R. L. (2003). Las estructuras elementales de la violencia: contrato y status en la etiología de la violencia (n. 334). Universidade de Brasília, Departamento de Antropología.

Uwineza, J., \& Brackelaire, J. L. (2014). Après le génocide, régénérer 1'“entre générations" pour naître à soi. Cahiers de psychologie clinique, 2, 143-171. 


\section{ARTIGOS}

\section{Resumenes}

(Efeitos da violência nos processos de historização: mediação simbólica, vínculo social e singularidade)

$O$ artigo aborda os efeitos da violência nos processos subjetivos de historização e no vínculo social. Com esse objetivo é desenvolvido um estudo teórico que integra reflexões psicanalíticas e hipóteses clínicas e filosóficas sobre a violência nas sociedades contemporâneas. Sustenta-se que a elaboração dos efeitos da violência depende da construção das mediações simbólicas que permitem a inscrição e a singularização da história subjetiva no vínculo social.

Palavras-chave: Violência, historização, ordem simbólico, singularidade

(The effects of violence on historization processes: symbolic mediation, social ties and singularity)

This paper analyzes the effects of violence on the subjective processes of historization and on social ties. To achieve that goal, a theoretical study was conducted that included psychoanalytical reflections, as well as clinical and philosophical hypotheses on violence in contemporary societies. We conclude that processing effects of violence depends on the construction of symbolic mediations that allow the inscription and singularization of subjective history in social ties.

Key words: Violence, historization, symbolic order, singularity

(Effets de la violence dans les processus d'historisation: médiations symbolique, lien social et singularité)

Cet article discute les effets de la violence sur les processus subjectifs de l'historicisation et sur le lien social. À fin d'atteindre cet objectif, une étude théorique a été développée qui intégrait des réflexions psychanalytiques ainsi que des hypothèses cliniques et philosophiques sur la violence dans les sociétés contemporaines. On fait valoir que l'élaboration des effets de la violence dépend de la construction de médiations symboliques qui permettent l'inscription et la singularisation de l'histoire subjective dans le lien social.

Mots clés: Violence, historicisation, ordre symbolique, singularité

(Auswirkungen von Gewalt auf die Prozesse der Historisierung: symbolische Vermittlung, soziale Bindung und Singularität)

Dieser Artikel untersucht die Auswirkungen von Gewalt auf die subjektiven Prozesse der Historisierung und auf soziale Bindungen. Zu diesem Zweck wurde eine theoretische Studie durchgeführt, die psychoanalytische Betrachtungen sowie klinische und philosophische Hypothesen zur Gewalt in zeitgenössischen 
Gesellschaften beinhaltete. Es wird die Ansicht vertreten, dass die Verarbeitung der Auswirkungen von Gewalt von der Konstruktion symbolischer Vermittlungen abhängt, die die Integrierung und Singularisierung der subjektiven Geschichte in soziale Bindungen erlauben.

Schlüsselwörter: Gewalt, Historisierung, symbolische Ordnung, Singularität

Citação/Citation: Jofré, D. (2018, junho). Efectos de la violencia en los procesos de historización: mediación simbólica, vínculo social y singularidad. Revista Latinoamericana de Psicopatologia Fundamental, 21(2), 293-308. http://dx.doi.org/10.1590/1415-4714.2018v$2 \ln 2 \mathrm{p} 293.5$.

Editores do artigo/Editors: Profa. Dra. Ana Maria Rudge e Profa. Dra. Sonia Leite.

Recebido/Received: 18.11.2017/ 11.18.2017 Aceito/Accepted: 15.2.2018 / 2.15.2018

Copyright: (C) 2009 Associação Universitária de Pesquisa em Psicopatologia Fundamental/ University Association for Research in Fundamental Psychopathology. Este é um artigo de livre acesso, que permite uso irrestrito, distribuição e reprodução em qualquer meio, desde que o autor e a fonte sejam citados / This is an open-access article, which permits unrestricted use, distribution, and reproduction in any medium, provided the original authors and sources are credited.

Financiamento/Funding: O autor declara não ter sido financiado ou apoiado / The author has no support or funding to report.

Conflito de interesses/Conflict of interest: $\mathrm{O}$ autor declara que não há conflito de interesses

/ The author has no conflict of interest to declare.

\section{DANIEL JoFré}

Docteur en Recherches en Psychanalyse et Psychopathologie, Université Paris 7-Diderot (Paris. França); Profesor de Psicopatología y de Psicología clínica, Universidad Austral de Chile (Puerto Montt, Chile).

Universidad Austral de Chile

Casilla N 1327

Puerto Montt, Chile

jofre.daniel@gmail.com / daniel.jofre@uach.cl

This is an open-access article, which permits unrestricted use, distribution, the original authors and sources are credited. 